\title{
Enantioselective synthesis of lepadins A-D from a phenylglycinol-derived hydroquinolone lactam
}

\author{
Mercedes Amat*, Alexandre Pinto, Rosa Griera, and Joan Bosch*
}

\begin{abstract}
The marine alkaloids (-)-lepadins A-C and (+)-lepadin D, belonging to two diastereoisomeric series, were synthesized from an $(R)$-phenylglycinol-derived tricyclic lactam via a common cisdecahydroquinoline intermediate. Crucial aspects of the synthesis are the stereochemical control in the assembly of the cisdecahydroquinoline platform, in the introduction of the C-2 methyl and C-3 hydroxy substituents, and in the generation of the C-5 stereocenter.
\end{abstract}

\section{Introduction}

Decahydroquinoline (DHQ) alkaloids constitute an important group of natural products, which exhibit a wide range of biological activities. Rather than in plants, they occur mainly in other terrestrial (amphibians, arthropods) and marine (tunicates, flatworms) organisms. ${ }^{[1]}$ Most of these alkaloids are 2,5disubstituted DHQs (for instance, pumiliotoxin C, the most representative member of this group), but they also include 5,7disubstituted (the phlegmarine-type Lycopodium alkaloids) ${ }^{[2]}$ and 2,3,5-trisubstituted derivatives (lepadins). The latter are a relatively small group of cis-DHQ alkaloids isolated from marine sources, characterized by a C-2 methyl substituent, a C-3 oxygenated (hydroxy or acyloxy) group, and a C-5 functionalized eight-carbon chain. From the stereochemical standpoint, lepadins can be classified into three series, as shown in Figure 1.

The first isolation of a member of this group, lepadin A, was reported by Steffan in $1991^{[3]}$ from the tunicate Clavelina lepadiformis. A few years later, Andersen et al. described the isolation of lepadins $\mathrm{A}-\mathrm{C}$ from the flatworm Prostheceraeus villatus and its prey $C$. lepadiformis. ${ }^{[4]}$ Lepadins A and B were found to exhibit significant in vitro cytotoxicity against several human cancer cell lines. Then in 2002, Wright and coworkers reported the isolation of lepadins $\mathrm{D}-\mathrm{F}$ from the tunicate Didemnum sp..$^{[5]}$ and Carroll et al., lepadins $\mathrm{F}-\mathrm{H}$ from the Australian ascidian Aplidium tabascum. ${ }^{[6,7]}$ Lepadins D-F possess significant and selective antiplasmodial and antitrypanosomal actvivity. Their low cytotoxicity makes them attractive models for the development of new antimalarial agents.

[a] Prof. Dr. M. Amat, A. Pinto, Dr. R. Griera, Prof. Dr. J. Bosch Laboratory of Organic Chemistry

Faculty of Pharmacy and Institute of Biomedicine (IBUB)

University of Barcelona

08028 Barcelona (Spain)

Fax: (+34) 93-402-45-39

E-mail: joanbosch@ub.edu

Homepage: http://www.ub.edu/sintefarma/

Supporting information for this article is given via a link at the end of the document.

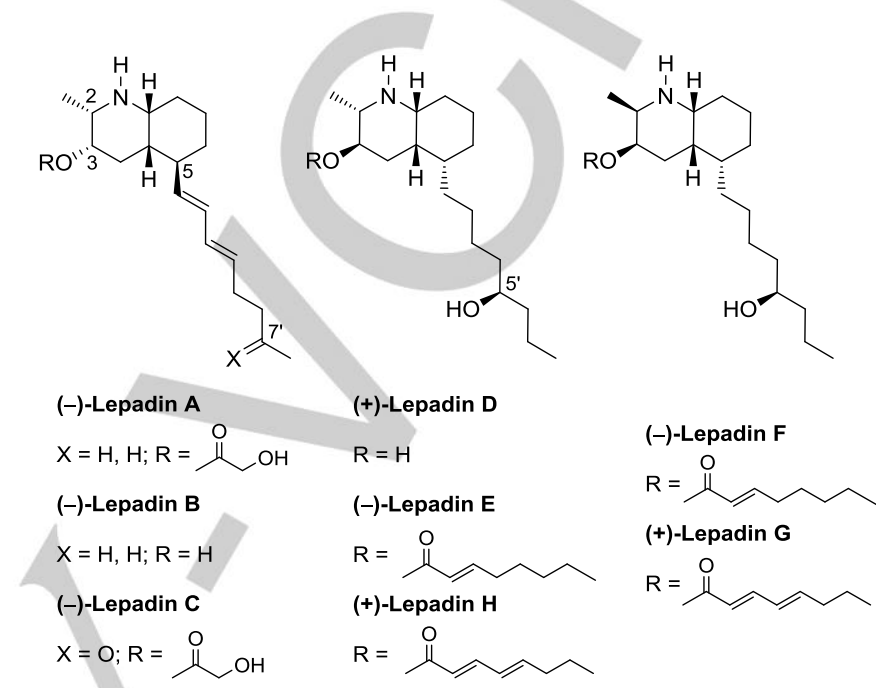

Figure 1. Lepadin alkaloids.

More recently, lepadin B was found to block neuronal nicotinic acetylcholine receptors. ${ }^{[8]}$ However, extensive bioactivity studies with lepadin alkaloids have been thwarted by the minute amounts isolated from natural sources.

Several enantioselective total syntheses of lepadins have been reported so far, ${ }^{[9,10]}$ which have allowed the relative and absolute configuration of these alkaloids to be unequivocally established. All these syntheses involve two phases: i) construction of a protected 3-hydroxy-2-methyl-cishydroquinoline platform, either functionalized at C-5 or bearing an oxidized one-carbon substituent at this position, and ii) installation of the appropriate C-5 eight-carbon chain. A variety of methodologies have been developed for the first phase: closure of the carbocyclic ring by aldol cyclization ${ }^{[9 a, b]}$ or a ringclosing/ring-opening metathesis sequence; ${ }^{; 9 d]}$ closure of the piperidine ring by intermolecular [3+3]-azacycloaddition, ${ }^{[9 f, g, 11]}$ xanthate-mediated free radical cyclization, ${ }^{[10]}$ or alkylative cyclization processes; ${ }^{[9 c]}$ and sequential one-pot construction of both rings from an acyclic precursor by a tandem ene-yne-ene ring-closing metathesis. ${ }^{[9 e]}$

On the other hand, different strategies have been used to assemble the eight-carbon chain at the DHQ C-5 position: i) starting from a C-5 unsubstituted $\mathrm{DHQ}$, direct introduction of an eight-carbon chain $\left(\mathrm{C}_{0}+\mathrm{C}_{8}\right.$ strategy) ${ }^{\left[{ }^{[9]}\right]}$ or sequential introduction of either one carbon and a seven-carbon chain $\left(\mathrm{C}_{0}+\mathrm{C}_{1}+\mathrm{C}_{7}\right)^{[9 \mathrm{a}, \mathrm{c}]}$ or two and six carbons $\left(\mathrm{C}_{0}+\mathrm{C}_{2}+\mathrm{C}_{6}\right)$; $\left.{ }^{[9 \mathrm{c}, \mathrm{f}, \mathrm{g}]} \mathrm{ii}\right)$ starting from a $\mathrm{DHQ}$ bearing a functionalized one-carbon substituent at $\mathrm{C}-5$, direct elongation of a seven-carbon chain $\left(\mathrm{C}_{1}+\mathrm{C}_{7} \text { strategy }\right)^{[9 \mathrm{e}]}$ or sequential elongation of one carbon and a six-carbon chain $\left(\mathrm{C}_{1}+\mathrm{C}_{1}+\mathrm{C}_{6}\right) \cdot{ }^{[9 b]}$ 


\section{Results and Discussion}

In previous work, we have developed straightforward procedures for the enantioselective synthesis of cis-DHQs bearing substituents at the 2-, 5-, and 6-positions, for instance the dendrobatid alkaloid (-)-pumiliotoxin C, using phenylglycinolderived oxazoloquinolone lactams $\mathbf{A}$ as enantiomeric scaffolds $^{[12]}$ (Scheme 1). These lactams are easily accessible by a stereoselective cyclocondensation reaction between $(R)$ phenylglycinol and appropriate cyclohexenone-derived $\delta$-keto esters. ${ }^{[13]}$

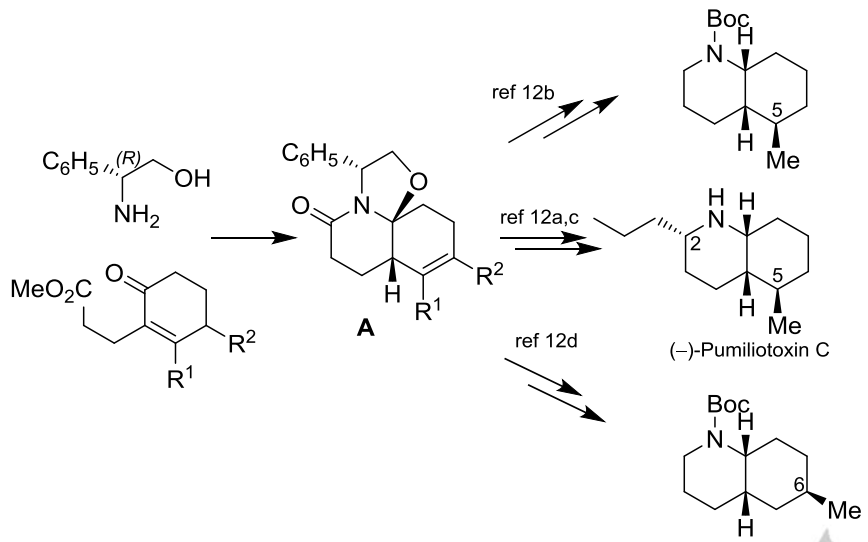

Scheme 1. Enantioselective access to substituted cis-decahydroquinolines from oxazoloquinolone lactams.

We envisaged tricyclic lactams $\mathbf{A}$ as suitable synthetic precursors of lepadins $A-C$ and $D, E, H$, belonging to two different stereochemical series. Starting from a lactam $\mathbf{B}\left(\mathbf{A} ; \mathbf{R}^{1}\right.$ $=\mathrm{CH}_{2} \mathrm{OProt}, \mathrm{R}^{2}=\mathrm{H}$ ), bearing an oxidized one-carbon substituent at the quinoline C-5 position, after stereoselective hydrogenation of the $\mathrm{C}-\mathrm{C}$ double bond and reductive removal of the oxazolidine ring, the stereoselective introduction of the methyl and hydroxy substituents, taking advantage of the lactam functionality, would give rise to a cis- $\mathrm{DHQ}$ C, which was visualized as a common intermediate en route to lepadins $A-E$ and $\mathrm{H}$ (Scheme 2).

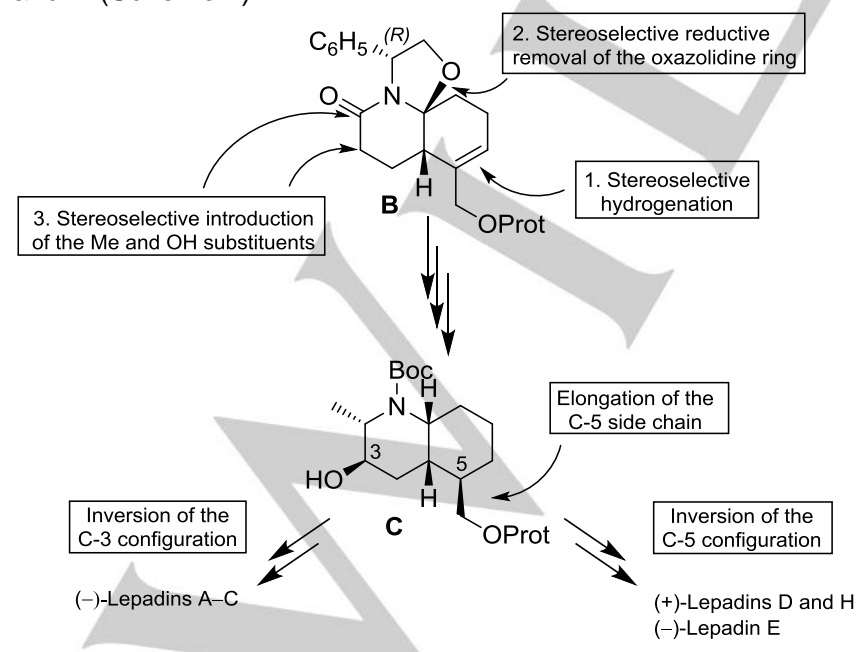

Scheme 2. Synthetic strategy.
The synthesis of (-)-lepadins $A-C$ from $\mathbf{C}$ would involve the inversion of the configuration of the $\mathrm{DHQ}$ C-3 position and the elongation of the C-5 side chain. ${ }^{[14]}$ In turn, an inversion of the configuration at C-5 followed by the elongation of the C-5 chain would afford (+)-lepadin D and their acylated derivatives (-)lepadin $\mathrm{E}$ and $(+)$-lepadin $\mathrm{H}$.

Scheme 3 outlines the initial steps of the synthesis. The required $\delta$-keto ester 3 , bearing a trimethylsilylethyl (TMSE)protected hydroxymethyl substituent, was prepared in two steps from diketo ester $\mathbf{1}$, by Pd-catalyzed cross-coupling of either the corresponding vinyl triflate or, more conveniently, bromo enone with potassium alkoxymethyltrifluoroborate $\mathbf{2}^{[15]}$ (Scheme 3 ).

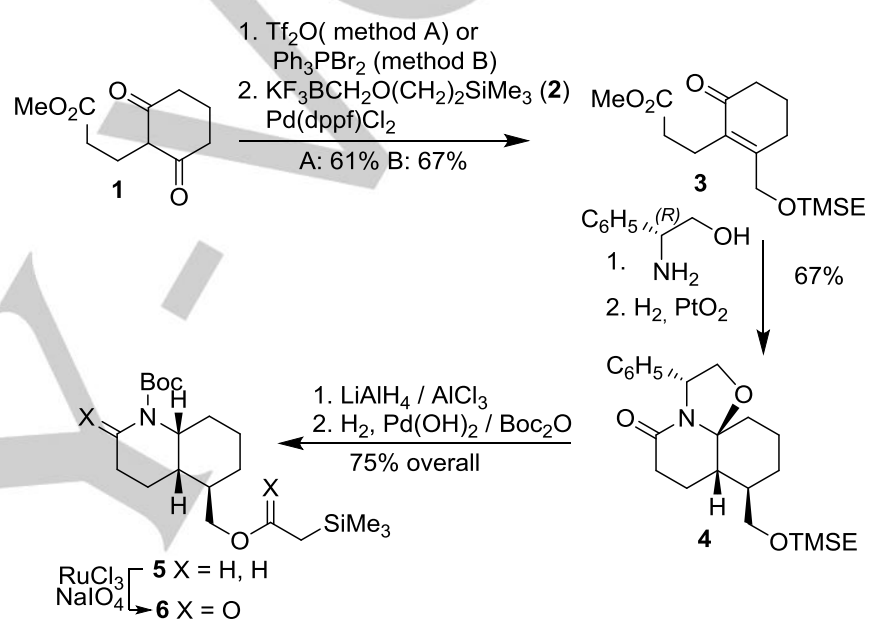

Scheme 3. Initial steps of the synthesis.

A cyclocondensation reaction of $\delta$-keto ester 3 with $(R)$ phenylglycinol, followed by catalytic hydrogenation of the resulting mixture of endocyclic and exocyclic unsaturated lactams led to saturated tricyclic lactam 4 in $67 \%$ overall yield. ${ }^{[16]}$

The reductive opening of the oxazolidine ring was accomplished with complete retention of configuration by alane reduction, which also caused the reduction of the lactam carbonyl. A subsequent hydrogenolysis of the benzylic $\mathrm{C}-\mathrm{N}$ bond in the presence of $\mathrm{Boc}_{2} \mathrm{O}$ stereoselectively gave cis-DHQ 5 in $75 \%$ overall yield. Although the use of the above sequence requires an additional oxidation step to recover the lactam functionality, it ensures the cis DHQ ring fusion characteristic of lepadins. ${ }^{[17]}$ The lactam carbonyl was efficiently reinstalled by ruthenium-promoted oxidation, ${ }^{[18]}$ although these conditions unexpectedly caused the concomitant oxidation of the silylethoxy moiety to give lactam ester $\mathbf{6}$.

To overcome this inconvenience, the TMSE protecting group was replaced by TIPS ${ }^{[19]}$ as shown in Scheme 4 and, satisfactorily, the ruthenium-mediated oxidation of the TIPSsubstituted DHQ 7 afforded lactam 8 in nearly quantitative yield.

Following our synthetic plan, the next step of the synthesis was the stereoselective introduction of the C-2 methyl and C-3 hydroxy substituents. This was achieved in three steps, via the enecarbamate 10, which was prepared by treatment of lactam 8 


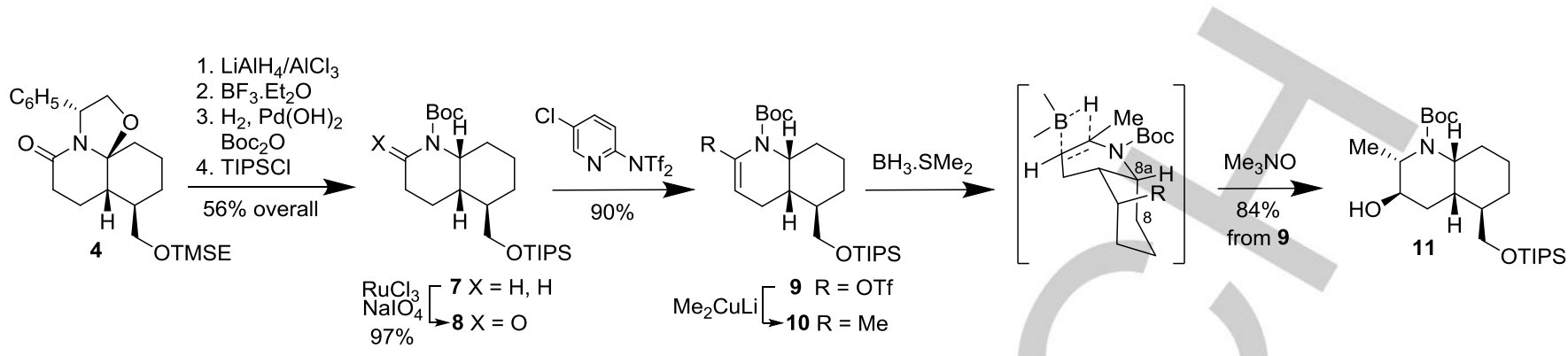

Scheme 4. Access to the advanced common intermediate 11.

with Comins' triflating reagent ${ }^{[20]}$ followed by reaction of the resulting vinyl triflate 9 with $\mathrm{Me}_{2} \mathrm{CuLi}^{[21]}$ (Scheme 4).

A subsequent hydroboration-oxidation sequence using $\mathrm{BH}_{3} \cdot \mathrm{SMe}_{2}$ complex ${ }^{[22]}$ and trimethylamine $\mathrm{N}$-oxide ${ }^{[2,23]}$ stereoselectively provided the C-2/C-3 trans alcohol 11 (C, Prot $=$ TIPS) in $84 \%$ overall yield from 9 . The formation of a single stereoisomer in the process is a consequence of borane approaching the most accessible face of the double bond of $\mathbf{1 0}$, which adopts a conformation with an axial $\mathrm{C}_{8}-\mathrm{C}_{8 \mathrm{a}}$ bond to avoid the $A^{(1,3)}$ strain caused by the Boc substituent.

The inversion of the $\mathrm{C}-3$ configuration, required for the synthesis of lepadins A-C from intermediate 11, was accomplished in $84 \%$ yield by Dess-Martin oxidation followed by $\mathrm{NaBH}_{4}$ reduction of the resulting ketone 12, which occurred stereoselectively from the less hindered face of the carbonyl group (Scheme 5). After protection of the C-3 hydroxy group of 13 as an acetate, and conversion of the protected hydroxymethyl substituent into a formyl group, the required $E, E$ configurated eight-carbon chain at C-5 was directly assembled, in a stereoselective manner, from aldehyde $\mathbf{1 4}$ by the HornerWadsworth-Emmons methodology using the anion derived from diethyl $(E)$-hept-2-enylphosphonate $(15)\left(\mathrm{C}_{1}+\mathrm{C}_{7}\right.$ strategy).<smiles>C[C@H]1C(=O)C[C@H]2[C@@H](C[O-])CCC[C@]2(C(=O)O)N1C(=O)[O-]</smiles>

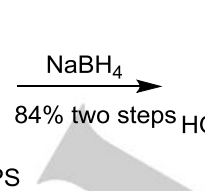

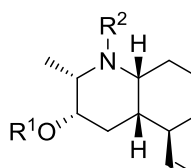

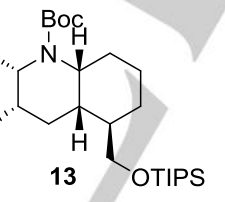

13 1. $\mathrm{Ac}_{2} \mathrm{O}$ 2. TBAF<smiles>[R10]CCCC(C)CC=CC=CCCCC</smiles>

ref 9b,c $\left[\begin{array}{l}16 \mathrm{R}^{1}=\mathrm{H}, \mathrm{R}^{2}=\mathrm{Boc} \\ (-) \text {-Lepadin } B \mathrm{R}^{1}=\mathrm{R}^{2}=\mathrm{H}\end{array}\right] \mathrm{TFA}$ (-)-Lepadin $\mathrm{AR}^{1}=\mathrm{COCH}_{2} \mathrm{OH}$ $R^{2}=\mathrm{H}$
Diene 16, in which deprotection of the C-3 hydroxy group had occurred, was obtained in $52 \%$ overall yield from 13. A final removal of the Boc protecting group gave (-)-lepadin B. The synthesis of $\mathbf{1 6}$ also constitutes a formal total synthesis of (-)lepadin $A^{[9 b, c]}$

The NMR data of our synthetic (-)-lepadin B were coincident with those reported in the literature, while its specific rotation was in good agreement with the value reported for this compound. ${ }^{[9 a]}$ The synthesis of (-)-lepadin B consists of 19 synthetic steps from $\delta$-keto ester 3 and takes place in $7.6 \%$ overall yield, which represents a significant improvement with regard to previous syntheses of this alkaloid.

Starting from the above aldehyde 14, a similar HornerWadsworth-Emmons reaction using the functionalized phosphonate 17 , led to 18 , a known precursor ${ }^{[9 c]}$ of $(-)$-lepadin $C$ (Scheme 6)

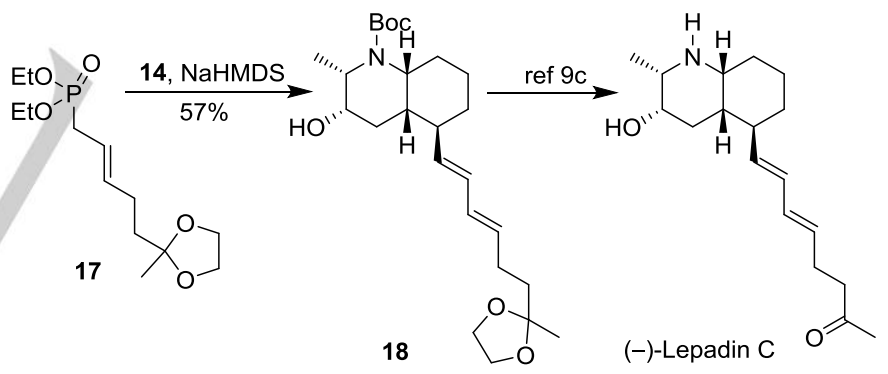

Scheme 6. Elongation of the C-5 side chain. Enantioselective synthesis of (-)lepadin C.

The key step to access lepadin D, and the configurationally related lepadins $\mathrm{E}$ and $\mathrm{H}$, from the common intermediate 11 was the inversion of the C-5 configuration. To accomplish this, we envisaged a synthetic sequence involving the stereoselective hydrogenation of a substituted $\mathrm{DHQ}$, such as $20 \mathrm{a}$, bearing an exocyclic double bond at the C-5 position. In this way, the eightcarbon chain would be assembled in two phases $\left(\mathrm{C}_{1}+\mathrm{C}_{2}+\mathrm{C}_{5}\right.$ strategy). Following the same three-step sequence used for the above conversion $13 \rightarrow \mathbf{1 4}$, alcohol 11 was converted to aldehyde 19a in excellent overall yield (Scheme 7). 

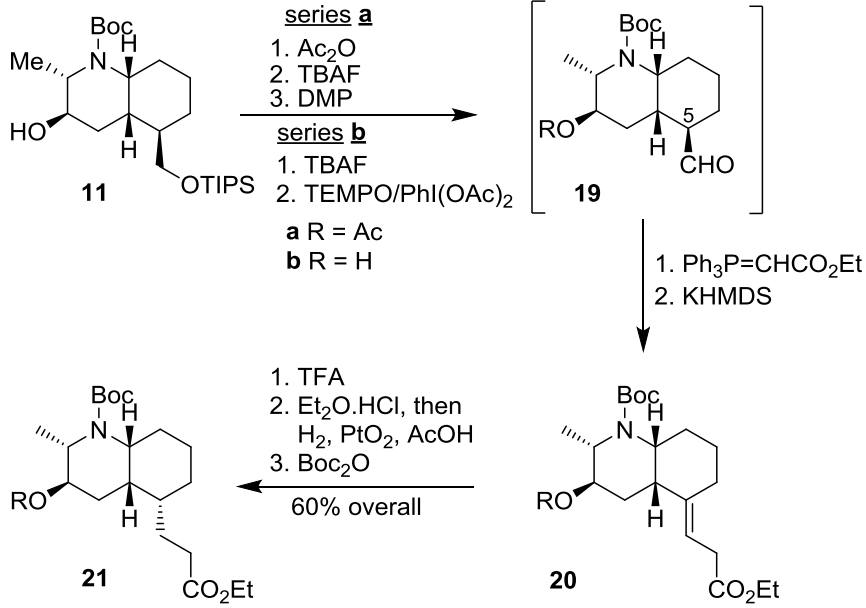

From 11: $46 \%(20 a) ; 59 \%(20 b)$

Scheme 7. Inversion of the C-5 configuration and two-carbon elongation.

An initial two-carbon elongation by Wittig olefination with (ethoxycarbonylmethylene)triphenylphosphorane, followed by base-promoted isomerization of the resulting conjugated carbon-carbon double bond to an exocyclic position ${ }^{[24]}$ led to unsaturated ester 20a. ${ }^{[25,26]}$ After removal of the Boc protecting group, ${ }^{[27]}$ catalytic hydrogenation of the resulting secondary amine (as hydrochloride ${ }^{[28]}$ ) in $\mathrm{AcOH}$ stereoselectively installed the required $\mathrm{C}-5$ stereochemistry. A subsequent protection with $\mathrm{Boc}_{2} \mathrm{O}$ afforded 21a. ${ }^{[29]}$

A notable improvement in terms of simplicity and chemical yield was achieved when the above sequence was directly carried out without protecting the C-3 hydroxy group, and the TEMPO/Phl $(\mathrm{OAc})_{2}$ system was used in the oxidizing step in a one-pot oxidation-olefination process. ${ }^{[30]}$ Thus, removal of the TIPS protecting group of $\mathbf{1 1}$, followed by chemoselective oxidation of the primary alcohol function with TEMPO and in situ Wittig homologation of the resulting aldehyde $19 \mathrm{~b}$, gave an $\alpha, \beta-$ unsaturated ester, which was converted to DHQ-5-propionate 21b via olefinic ester $\mathbf{2 0 b}$, as in the above a series.

To complete the functionalized eight-carbon chain of $(+)$ lepadin $D$ from the advanced intermediate $\mathbf{2 1 b}$, it was necessary to perform a five-carbon elongation. After protection of the C-3 hydroxy group as a TBDMS ether, $\mathrm{LiAlH}_{4}$ reduction of the ester group, followed by conversion of the resulting alcohol to an iodide using the $\mathrm{I}_{2}-\mathrm{PPh}_{3}$-imidazole protocol and subsequent reaction with sodium benzenesulfinate gave sulfone $\mathbf{2 2}$ (Scheme 8).

Coupling of the sulfonyl carbanion derived from 22 with the easily accessible epoxide $(R)$-propyloxirane ${ }^{[31]}$ gave hydroxy sulfone 23 (mixture of epimers) in 60\% yield. Finally, reductive desulfonylation with sodium amalgam, followed by simultaneous removal of the Boc and silyl protecting groups, completed the synthesis of $(+)$-lepadin $D$, in a total of 25 steps and $1.4 \%$ overall yield. Both the specific rotation and NMR data of our synthetic (+)-lepadin D were consistent with those reported for the natural product. ${ }^{[5]}$

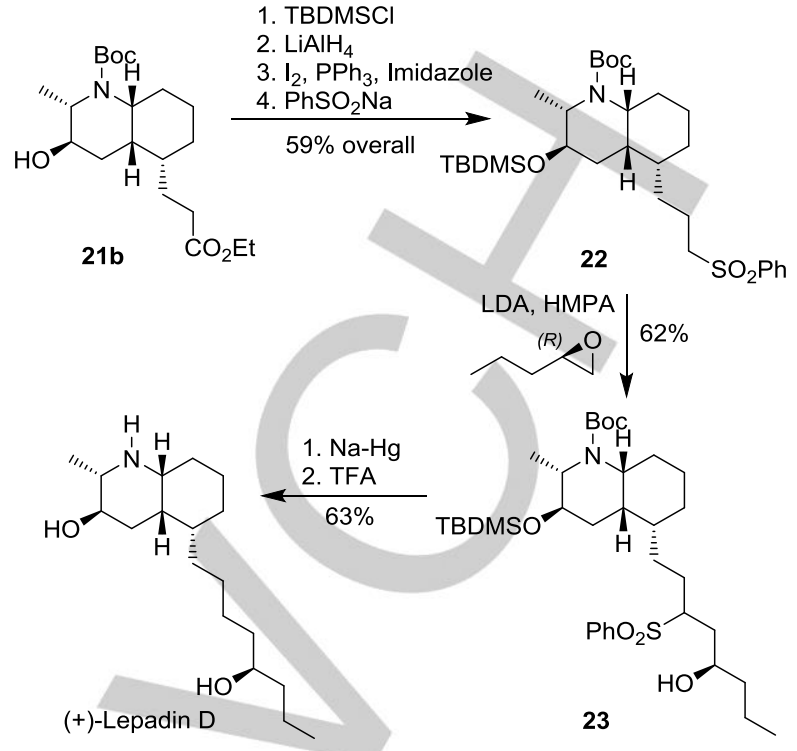

Scheme 8. Completion of the C-5 side chain. Enantioselective synthesis of (+)-lepadin D.

\section{Conclusions}

In conclusion, enantioselective synthetic routes to two stereochemical groups of lepadins have been developed from a common $\mathrm{DHQ}$ intermediate. The synthesis relies on the versatility of phenylglycinol-derived lactams as multipurpose scaffolds for the enantioselective construction of complex nitrogen-containing natural products.

The main features of the synthesis are: i) the straightforward construction of a DHQ system bearing a functionalized onecarbon substituent at the C-5 position; ii) the incorporation of the C-2 methyl and C-3 hydroxy substituents taking advantage of the lactam carbonyl; and iii) the elongation of the C-5 chain, either directly with a seven-carbon fragment (lepadins A-C) or sequentially using two- and five-carbon fragments (lepadin D).

Key aspects from the stereochemical standpoint are: i) the stereoselective generation of the cis-DHQ ring fusion by reductive opening of the oxazolidine ring; ii) the control of the configuration of the C-5 stereocenter by stereoselective hydrogenation of a carbon-carbon double bond, either endocyclic in a tricyclic lactam or exocyclic in an $\mathrm{N}$-unsubstituted $\mathrm{DHQ}$ derivative; iii) the installation of the trans $2-\mathrm{Me} / 3-\mathrm{OH}$ relationship by a stereoselective hydroboration-oxidation sequence from a methyl-substituted $\mathrm{N}$-Boc enamine, with subsequent inversion of the $\mathrm{C}-3$ configuration via a ketone; and, finally, iv) the stereoselective achievement of the $(E, E)$ configuration of the C-5 chain via a Horner-Wadsworth-Emmons reaction. 


\section{Acknowledgements}

Financial support from the Spanish Ministry of Economy and Competitiveness (Project CTQ2012-35250) and the AGAUR, Generalitat de Catalunya (Grant 2014SGR155) is gratefully acknowledged. Thanks are also due to the MINECO for a fellowship to A.P.

Keywords: alkaloids $\cdot$ asymmetric synthesis $\bullet$ lactams $\bullet$ nitrogen heterocycles $\bullet$ total synthesis

[1] (a) J. W. Daly, H. M. Garraffo, T. F. Spande, in Alkaloids: Chemical and Biological Perspectives, Vol. 13, (Ed.: S. W. Pelletier), Pergamon, New York, 1999, pp. 1-161; (b) T. F. Spande, P. Jain, H. M. Garraffo, L. K Pannell, H. J. C. Yeh, J. W. Daly, S. Fukumoto, K. Imamura, T. Tokuyama, J. A. Torres, R. R. Snelling, T. H. Jones, J. Nat. Prod. 1999 62, 5-21; (c) J. W. Daly, T. F. Spande, H. M. Garraffo, J. Nat. Prod. 2005, 68, 1556-1575. (d) For a review on the synthesis of DHQs see: C. Kibayashi, S. Aoyagi, in Studies in Natural Products Chemistry; Vol. 19, (Ed.: Atta-ur-Rahman), Elsevier, Amsterdam, 1997, pp. 3-88

[2] P. Siengalewicz, J. Mulzer and U. Rinner, in The Alkaloids, Vol. 72, (Ed.: H.-J. Knölker), Elsevier, San Diego, 2013, pp. 1-151.

[3] B. Steffan, Tetrahedron 1991, 47, 8729-8732.

[4] J. Kubanek, D. E. Williams, E. D. de Silva, T. Allen, R. J. Andersen, Tetrahedron Lett. 1995, 36, 6189-6192.

[5] A. D Wright, E. Goclik, G. M. König, R. Kaminsky, J. Med. Chem. 2002, 45, 3067-3072.

[6] R. A. Davis, A. R. Carroll, R. J. Quinn, J. Nat. Prod. 2002, 65, 454-457.

[7] Both dextrorotatory ${ }^{[6]}$ and levorotatory ${ }^{[5]}$ lepadin $F$ have been reported. However, the small values of the optical rotation and the fact that $(+)$ lepadin $\mathrm{F}$ was isolated as a red oil seem to suggest ${ }^{\left[{ }^{e e]}\right.}$ that natural lepadin $\mathrm{F}$ is levorotatory.

[8] H. Tsuneki, Y. You, N. Toyooka, T. Sasaoka, H. Nemoto, J. A. Dani I. Kimura, Biol. Pharm. Bull. 2005, 28, 611-614.

[9] (a) N. Toyooka, M. Okumura, H. Takahata, J. Org. Chem. 1999, 64, 2182-2183 [(-)-lepadin B]; (b) T. Ozawa, S. Aoyagi, C. Kibayashi, J. Org. Chem. 2001, 66, 3338-3347 [(-)-A-C]; (c) X. Pu, D. Ma, J. Org Chem. 2006, 71, 6562-6572 [(-)-A-C, E; (+)-D,H]; (d) G. Barbe, A Charette, J. Am. Chem. Soc. 2008, 130, 13873-13875 [(+)-B]; (e) A Niethe, D. Fischer, S. Blechert, J. Org. Chem. 2008, 73, 3088-3093 [(+)-F, (-)-G]; (f) G. Li, R. P. Hsung, B. W. Slafer, I. K. Sagamanova Org. Lett. 2008, 10, 4991-4994 [(+)-F]; (g) G. Li, R. P. Hsung, Org. Lett. 2009, 11, 4616-4619 [(+)-G]. (h) For a review, see: A. Pelss, A. M. P. Koskinen, Chem. Heterocycl. Compd. 2013, 49, 226-240.

[10] For a formal synthesis of ( \pm )-lepadin B, see: C. Kalaï, E. Tate, S. Z. Zard, Chem. Commun. 2002, 1430-1431.

[11] For a diastereodivergent approach to cis-DHQ precursors of lepadins using an aza-[3+3] annulation strategy, see: G. Li, L. J. Carlson, I. K. Sagamanova, B. W. Slafer, R. P. Hsung, C. Gilardi, H. M. Sklenicka, N. Sydorenko, Synthesis 2009, 2905-2914.

[12] (a) M. Amat, R. Griera, R. Fabregat, E. Molins, J. Bosch, Angew. Chem. 2008, 120, 3348-3351; Angew. Chem. Int. Ed. 2008, 47, 3348 3351; (b) M. Amat, R. Fabregat, R. Griera, J. Bosch, J. Org. Chem. 2009, 74, 1794-1797; (c) M. Amat, R. Fabregat, R. Griera, P. Florindo, E. Molins, J. Bosch, J. Org. Chem. 2010, 75, 3797-3805; (d) M. Amat, L. Navío, N. Llor, E. Molins, J. Bosch, Org. Lett. 2012, 14, 210-213.

[13] For a related procedure for the enantioselective synthesis of 8 substituted and 6,8-disubstituted cis-DHQs using cyclohexanonederived $\delta$-keto (di)ester derivatives, see: M. Amat, E. Ghirardi, L. Navío,
R. Griera, N. Llor, E. Molins, J. Bosch, Chem. Eur. J. 2013, 19, 1604416049.

[14] For a preliminary account of this part of the work, see: M. Amat, A Pinto, R. Griera, J. Bosch, Chem. Commun. 2013, 49, 11032-11034.

[15] (a) For the preparation of 2 and its cross-coupling reaction with aryl chlorides, see: G. A. Molander, B. Canturk, Org. Lett. 2008, 10, 21352138. (b) For related processes with alkenyl bromides and potassium alkyltrifluoroborates, see: G. A. Molander, J. Ham, D. G. Seapy, Tetrahedron 2007, 63, 768-775.

[16] Minor amounts of the epimer at the C-5 position $(<5 \%)$ and the diastereoisomer at the $4 \mathrm{a}, 5$, and 8 positions ( $20 \%$ ) of the $\mathrm{DHQ}$ ring were also isolated.

[17] Operating from a model tricyclic lactam bearing a methyl substituent instead of $\mathrm{CH}_{2} \mathrm{OTMSE}$, the reductive cleavage of the oxazolidine $\mathrm{C}-\mathrm{O}$ bond using $\mathrm{Et}_{3} \mathrm{SiH}-\mathrm{TiCl}_{4}$ took place with moderate stereoselectivity, leading to a 5:1 mixture of cis-trans DHQs. ${ }^{[14]}$

[18] (a) J. C. Sheehan, R. W. Tulis, J. Org. Chem. 1974, 39, 2264-2267; (b) K. Moriyama, H. Sakai, T. Kawabata, Org. Lett. 2008, 10, 3883-3886.

[19] The direct introduction of a TIPS-protected hydroxymethyl substituent using the Molander methodology is not possible because the lower nucleophilicity of silanols compared with alcohols prevents the preparation of the required trifluoroborate reagent $\mathrm{KBF}_{3} \mathrm{CH}_{2} \mathrm{OTIPS}$. ${ }^{15 \mathrm{a}]}$

[20] D. L. Comins, A. Dehghani, Tetrahedron Lett. 1992, 33, 6299-6302.

[21] (a) C. J. Foti, D. L. Comins, J. Org. Chem. 1995, 60, 2656-2657; (b) K. Tsushima, T. Hirade, H. Hasegawa, A. Murai, Chem. Lett. 1995, 801 802.

[22] L. Le Corre, J.-C. Kizirian, C. Levraud, J.-L. Boucher, V. Bonnet, H. Dhimane, Org. Biomol. Chem. 2008, 6, 3388-3398.

[23] (a) G. W. Kabalka, H. C. Hedgecock Jr., J. Org. Chem. 1975, 40, 1776 1779; (b) T. Luker, H. Hiemstra, W. N. Speckamp, J. Org. Chem. 1997, 62, 3592-3596; (c) L. Bartali, A. Casini, A. Guarna, E. G. Occhiato, D Scarpi, Eur. J. Org. Chem. 2010, 5831-5840.

[24] For a related Wadsworth-Emmons olefination-deconjugation sequence, see: L. A. Paquette, J. Tae, E. R. Hickey, W. E. Trego, R. D. Rogers, J. Org. Chem. 2000, 65, 9160-9171.

[25] Partial hydrolysis of the acetate function was observed. The resulting alcohol $20 \mathrm{~b}$ could be reacetylated to $20 \mathrm{a}$ with $\mathrm{Ac}_{2} \mathrm{O}$.

[26] The direct introduction of a functionalized seven-carbon chain $\left(C_{1}+C_{7}\right.$ strategy) by $B$-alkyl Suzuki-Miyaura coupling was not studied because the required vinyl triflate derived from $19 a$ was obtained in low yield.

[27] Due to conformational factors, the presence of an $N$-Boc substituent decreases the stereoselectivity of the catalytic hydrogenation of the exocyclic double bond in 5-alkylidene-DHQ derivatives: see ref 9c.

[28] Somewhat unexpectedly, the catalytic hydrogenation $\left(\mathrm{PtO}_{2}\right.$ or $\mathrm{Pt} / \mathrm{C}$, $\mathrm{MeOH}$ or $\mathrm{AcOH}$ ) of the free base was extremely slow and led to considerable amounts of the corresponding N-methyl-DHQ-5propionate.

[29] The stereochemical assignment of 21a was confirmed by comparing its ${ }^{13} \mathrm{C}$ NMR data [ $\delta 18.5$ (C-4), 53.0 and 54.2 (two rotamers, C-8a)] with those of 5-epi-21a [ $\delta 27.8(\mathrm{C}-4), 50.4(\mathrm{C}-8 \mathrm{a})$ ], which was prepared by direct catalytic hydrogenation of the Wittig adduct formed from 19a.

[30] J.-M. Vatèle, Tetrahedron Lett. 2006, 47, 715-718.

[31] R. Masuo, K. Ohmori, L. Hintermann, S. Yoshida, K. Suzuki, Angew. Chem. 2009, 121, 3462-3465; Angew. Chem. Int. Ed. 2009, 48, 3462 3465 . 


\section{FULL PAPER}

Using $(R)$-phenylglycinol as the source of chirality, the marine alkaloids (-)-lepadins A-C and (+)lepadin $\mathrm{D}$, with opposite absolute configuration at the C-3 and C-5 ring positions, have been synthesized. The synthesis features the use of a tricyclic oxazoloquinolone lactam as the key enantiomeric scaffold and proceeds via an advanced common decahydroquinoline intermediate.

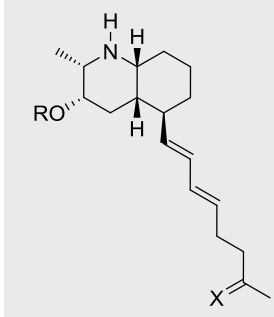

(-)-Lepadins A-C $\mathrm{R}=\mathrm{H}$ or $\mathrm{COCH}_{2} \mathrm{OH}$ $\mathrm{X}=\mathrm{H}, \mathrm{H}$ or $\mathrm{O}$
Mercedes Amat*, Alexandre Pinto, Rosa Griera, and Joan Bosch*

Page No. - Page No.

Enantioselective synthesis of lepadins A-D from a phenylglycinolderived hydroquinolone lactam 